\title{
Exploring the Use of Computer-aided Learning Modules (CAL) to Enhance the Teaching and Learning of Pharmacokinetics to Pharmacy Students
}

\author{
Mumtaz Hussain', Shariza Sahudin ${ }^{2, *}$, Izzati Yussof ${ }^{2}$ \\ 'Boots the Chemist, 12-18 Whitgift Centre, Croydon CRO 1SN, UK. \\ ${ }^{2}$ The Faculty of Pharmacy, Universiti Teknologi MARA, Puncak Alam Campus, Selangor, MALAYSIA.
}

\begin{abstract}
Objectives: To develop an integrated software package to augment the teaching and learning of pharmacokinetics to undergraduate pharmacy students, covering various aspects of pharmacokinetics from basic principles, calculations and their application, including therapeutic drug monitoring (TDM) in clinical practice and bioequivalence calculations for industry, as well as several computer-aided learning modules (CAL). Materials and Methods: JAVA was utilized to allow for a modular design and the ability to build future functionality into the system. A database of patient information with multiple drugs of interest, with plasma concentration values, was constructed to allow students to appreciate the variability of pharmacokinetic parameters in different clinical conditions. Computer-assisted learning (CAL) modules were prepared to assist students in understanding selected pharmacokinetics topics. Software testing, validation, system testing and user satisfaction surveys were conducted to evaluate suitability and accuracy. Results: A comprehensive and modular pharmacokinetics computer program was successfully developed using Java and NetBeans. The program produced accurate and reproducible values for numerous pharmacokinetic parameters, based on a user satisfaction survey, the average usability score of 68.8 indicating good
\end{abstract}

usability status among undergraduate students. Conclusion: The software is the first attempt to produce a comprehensive package with multiple points of calculation including compartmental and non-compartmental analysis, TDM and bioequivalence, as well as learning modules, all integrated into one environment suitable for both pharmacy students and pharmacists. End users were generally satisfied with the software and provided feedback and recommendations to further improvements with the ultimate aim of introducing the system to undergraduates for teaching purposes.

Key words: Pharmaceutics, Education, Pharmacokinetics, Pharmacy practice, Drug delivery.

Correspondence

Dr. Shariza Sahudin

Department of Pharmaceutics Universiti Teknologi MARA Puncak Alam Campus 42300 Selangor, MALAYSIA

Phone: +603-3258-4645

Email: shariza2280@uitm.edu.my

DOI: 10.5530/jyp.2020.12.91

\section{INTRODUCTION}

Pharmacokinetics is taught within the pharmacy curriculum with a focus on basic concepts and definitions, together with a description of the common mathematical models. A typical pharmacy curriculum introduces these basic concepts early during a course; however, the clinical importance ${ }^{1}$ and industrial relevance ${ }^{2}$ of the subject, with an application to therapeutic drug monitoring (TDM), formulation design and regulatory implications are generally taught later in the final years. Separation of theory and application in this way may cause difficulty among some students to appreciate the clinical significance behind the equations presented. Thus it is important to better integrate these aspects of theory and application to prepare students for the clinical and industrial scenarios. For clinical pharmacy, for instance, mastery of pharmacokinetics is crucial since it is used to estimate optimal drug dosage regimens in different therapeutic situations, specifically in the context of TDM. Better knowledge and understanding of pharmacokinetics may also play a role in the avoidance of medication errors due to inappropriate dosage recommendations, which can either occur due to overdosing or underdosing. The assumption is that inaccuracies in dosage calculations, due to lack of understanding of the underlying methodologies or tools used to calculate dosages, leads to errors. Thus an improved learning process, designed specifically for the newer generation of students, together with traditional learning methods, may improve understanding and the implication is, reduce calculation-based errors. In Malaysia for instance, 17,357 medication errors were reported between 2009 and 2012 and $76.1 \%$ of these were due to prescribing errors, which include errors in dosing. ${ }^{3}$ Errors concerning TDM for instance can occur in various forms: a clinician ordering TDM when it is not indicated, incorrect sampling times, errors in drug concentration analysis and in pharmaceutical terms, incorrect interpretation of data and the subsequent inappropriate dose recommendation. ${ }^{4}$

Pharmacokinetics is a subject that is often not well received by students due to its basis in mathematics and difficulty in linking basic concepts with clinical relevance. ${ }^{5,6}$ One way to tackle this issue is by incorporating innovation to improve students' understanding of the subject. Various strategies have been adopted to improve the learning experience, including pharmacokinetics-based online games to help student's link theory and application. ${ }^{7}$ Such E-learning enables students from geographically-diverse populations to pursue further education, using technology to allow team-based learning despite the distance. ${ }^{8}$ The use of pharmacokinetic-based computer programs may also help students to achieve a better comprehension of pharmacological concepts, visualize the effect of changing pharmacokinetic parameters in patients via corresponding time-concentration curves and allow them to have a better appreciation of computer utilization in clinical practice. ${ }^{9}$ The new 
generation of learners or 'millennials', may be able to learn better with computers than traditional lectures and currently represent the majority of enrolled undergraduate-students in colleges and universities. ${ }^{10}$ Such students are comfortable with multi-tasking and multimedia use and may have little tolerance for traditional modes of information exchange. A survey by Deloitte in 15 developed countries revealed that $85 \%$ of millennials had access to a laptop computer and $87 \%$ had access to a smartphone. ${ }^{11}$ Therefore, educators must evolve and innovate to better complement the new generation of learners as the technologies of computers and smartphones offer exciting opportunities to be utilized in pedagogy. It is important to state that the authors do not propose to replace traditional teaching methods with software. Rather, we feel the current methods used to teach pharmacokinetics require modification to cater to a new generation of students, with their more technologicallyfocused learning styles. Traditional teaching methods, ensure students are provided with a rigorous grounding in pharmacokinetic principles with the ability to conduct manual calculations. These, however, need to be augmented with a software-based approach to allow a visual reinforcement of many of the fundamental concepts and to provide learners with a visual validation of their calculations.

The current study attempts to develop a novel modular software system that can be used to enhance the teaching, learning and application of pharmacokinetics, for both students and practitioners, with a focus on the provision of calculations for important parameters that define their behaviors following drug administration, together with comprehensive TDM calculators for practitioners, bioavailability calculators for drug delivery applications as well as ComputerAided Learning (CAL) modules specifically for teaching and learning. Although pharmacokinetic software packages are widely available, most tend to focus on either specific TDM calculations, ${ }^{12}$ or pharmacokinetic modelling, ${ }^{13}$ with a small proportion focusing on bioequivalence. ${ }^{14}$ Commercial software is often expensive and not focused on the aspect of teaching and learning and are therefore unsuitable for our purposes. Thus the current system attempts to combine all these areas, including core pharmacokinetic parameter calculations with multidose, applications to TDM and bioavailability, together with e-learning modules to produce, unlike existing software, an all-encompassing system designed for teaching and learning. The inclusion of a pharmacokinetic database, constructed from real patients' data may help students to understand the variability in pharmacokinetic parameters of the same drug among patients with varying ages and health conditions.

\section{MATERIALS AND METHODS}

The program was written in the Java language, a general purpose, highlevel, object-oriented, cross-platform programming language developed by Sun Microsystems, using NetBeans IDE Version 8.2. Java was chosen as it allows portability between devices, allowing the same compiled source code to be installed on different operating systems and the CAL package was developed using Adobe Captivate 2017. One-compartment, linear pharmacokinetic calculations were utilized for IV bolus, IV infusion and oral dose modules and the modular approach to development meant this can be expanded at a later date to include multi-compartment models. At the time of writing, two-compartment calculations had been implemented for IV-bolus administration. Oral dose calculations focused on the 'methods of residuals' to separate absorption and elimination rate parameters with a built-in consistency check for the ratio between the two values. Again, the modular approach will allow the inclusion of 'Wagner-Nelson' methods for calculation of oral dose absorption rate constants if this internal consistency check fails. TDM calculations were written by following the Malaysian Ministry of Health's (MOH) TDM guidelines. This is a crucial point, as the software will not be placed on general release until it has been validated and approved by the MOH. The aim here is to prevent its use in a clinical setting, with particular reference to the TDM modules. Until then, it will only be available from University servers and will not be provided to students as a stand-alone application. The ultimate iteration in development will move to a web-based system, which is currently near completion. This will not require installation and user access will be controlled to ensure the system is not used inappropriately. The bioequivalence calculations were conducted based on noncompartmental analysis, using the area under the curve (AUC) and area under the first moment curves (AUMC) as a basis for parameter calculations. These were then used to calculate Mean Absorption Time (MAT) and Mean Residence Time (MRT), together with relative and absolute bioavailability the construction of the database involved data collection from literature as well as retrospective data from real patients (Table 1). Data collection was done in Hospital Putrajaya (HPJ) from November 2017 until December 2017 from the pharmacy department's TDM registry. Data recorded by the pharmacy department between 2015 and 2017 was used to construct the database. No patient identifiers were used and each patient was anonymized by being assigned a unique Patient ID. A registry that identifies patients to their respective Patient ID in the database was kept separately in a password-protected file and will be kept for two years after study completion. The research needed to be registered with the National Medical Research Registry (NMRR) as it involved data collection from a Ministry of Health $(\mathrm{MOH})$ facility and ethics approval was obtained before data collection from the Universities Research Ethics Committee (REC/381/17). The study population for the database was calculated using Krejcie and Morgan ${ }^{15}$ where the population size was based on the population of Wilayah Persekutuan Kuala Lumpur, 2012. ${ }^{16}$ The target sample size was approximately 384 and this was rounded up to 400 , divided between the three drugs for the TDM modules, with 200 for gentamicin and 100 for vancomycin and phenytoin each. This division was based on the availability of data from the HPJ TDM Registry.

Table 1: List of Data Collected for Database.

\begin{tabular}{cccc}
\hline Parameters & Gentamicin & Vancomycin & Phenytoin \\
\hline Patient Weight & $\sqrt{ }$ & $\sqrt{ }$ & $\sqrt{ }$ \\
Patient Height & $\sqrt{ }$ & $\sqrt{ }$ & $\sqrt{ }$ \\
Patient Age & $\sqrt{ }$ & $\sqrt{ }$ & $\sqrt{ }$ \\
For Neonates: Gestational Age & $\sqrt{ }$ & $\sqrt{ }$ & \\
Patient Gender & $\sqrt{ }$ & $\sqrt{ }$ & $\sqrt{ }$ \\
Patient Race & $\sqrt{ }$ & $\sqrt{ }$ & $\sqrt{ }$ \\
CrCl & $\sqrt{ }$ & $\sqrt{ }$ & $\sqrt{ }$ \\
Plasma Creatinine & $\sqrt{ }$ & $\sqrt{ }$ & $\sqrt{ }$ \\
Albumin & & & $\sqrt{ }$ \\
Dose & $\sqrt{ }$ & $\sqrt{ }$ & $\sqrt{ }$ \\
Time of Dose & $\sqrt{ }$ & $\sqrt{ }$ & $\sqrt{ }$ \\
C $_{\mathrm{p}}$ versus Time & $\sqrt{ }$ & $\sqrt{ }$ & $\sqrt{ }$ \\
$\mathrm{K}_{\mathrm{e}}$ & $\sqrt{ }$ & $\sqrt{ }$ & $\sqrt{ }$ \\
$\mathrm{t}_{1 / 2}$ & $\sqrt{ }$ & $\sqrt{ }$ & $\sqrt{ }$ \\
$\mathrm{V}_{\mathrm{d}}$ & $\sqrt{ }$ & $\sqrt{ }$ & $\sqrt{ }$ \\
Diagnosis & $\sqrt{ }$ & $\sqrt{ }$ & $\sqrt{ }$ \\
Co-morbidities & $\sqrt{ }$ & $\sqrt{ }$ & $\sqrt{ }$ \\
Concomitant Drug & $\sqrt{ }$ & $\sqrt{ }$ & $\sqrt{ }$ \\
\hline
\end{tabular}


The program was validation by performing unit, usability and enduser testing. Unit testing of functions was conducted from the early stage of software development and was done on each function upon completion. Completed modules were combined and then integration tested, evaluating the communication between modules and the database. Test cases were prepared for each level of testing to analyze each function and integrated modules during development. Validation of modules was conducted by comparing output from the Software with manual calculations and graphically using Excel. A comparison of the Elimination rates produced, $\mathrm{K}_{\mathrm{e}}$, with a series of randomly selected values from HPJ's TDM Registry for the TDM Modules was also made. The analysis was conducted to evaluate the significance of any variations ${ }^{18}$ using a paired $t$-test, to ensure there was no significant difference in the mean output and a Bland-Altman plot used to determine the mean differences and constructing a limit of agreement ${ }^{18}$ from a standard deviation of mean $\pm 1.96 \mathrm{SD}$. This provided a simple way to evaluate any bias between the mean differences. Independent System Testing was also conducted by two software testers not involved in the development of the project, a certified software tester from the College of Computer Science and Information Technology, Universiti Tenaga Nasional and a practicing pharmacist with experience in conducting functionality test for Pharmacy Information Systems (PhIS).

To assess usability and user satisfaction, a user satisfaction survey was conducted among end-users via a validated three-section questionnaire. The sample size of 170 was calculated using the Krejcie and Morgan ${ }^{15}$ equation, of third-year undergraduate Universiti Teknologi MARA (UiTM) pharmacy students. However, several non-student individuals including practicing pharmacists, provisionally registered pharmacists (PRP) and one lecturer were also included for academic purposes, however without any statistical foundation. The target population was allowed to try using the Software through a standardized assignment or exercise, in which students were given pharmacokinetic and TDMrelated questions that needed answering with the help of the Software. The first section of the questionnaire collected basic demographic data, followed by section-two consisting of a validated 10-item, 5-point Likert System Usability Scale (SUS) developed by John Brooke for Digital Equipment Corporation $\odot$ that gives a global view of subjective assessments of software usability. ${ }^{19}$ The scoring for SUS is based on a single number ranging from 0 to 100 and a total score of 68 and above is generally considered to be above average and the cut-off point for acceptable usability. ${ }^{19}$ The third-section was used to measure user satisfaction in terms of GUI, output, the CAL Module, graphics and sound used, as well as general acceptability. The items were based on commonly-used themes found in other user experience surveys, such as efficiency, attractiveness, dependability and usefulness. ${ }^{20,21}$ The reliability of a questionnaire is measured based on the value of Cronbach's alpha; Cronbach's alpha of 0.7 and more is considered to have good reliability. ${ }^{22}$ Based on the pilot study result, a Cronbach's alpha of 0.863 was obtained, indicating good reliability. Spearman's rank-order correlation analysis was used to analyze the correlation between computer skills and usability scores. Spearman Rho's is a non-parametric test that can be used to analyse ordinal data.

\section{RESULTS}

The main menu of the program is invoked when the program is started and contains buttons that link to different functions (Figure 1). Functions under the 'Automated Graphs' require the input of time versus plasma concentration of a drug, together with the dose and units. Users can then generate corresponding graphs and obtain relevant pharmacokinetic values such as $\mathrm{Ke}_{1 / 2}, \mathrm{~V}_{\mathrm{d}}$, bioavailability. The GUI for IV Bolus, IV Infusion and Oral Dose Module input windows are very

similar and shown in Figure 2A. Users can input up to 10 values of time and plasma concentration, with a minimum of four values. The four values are needed to divide the graph into absorption (which essentially also involves distribution and elimination) and elimination phase. The output opens in a new window (Figure 2B). The Absolute and Relative Bioequivalence Modules are designed to compare the bioavailability of an oral and IV formulations, or two different oral formulations. The Pharmacokinetic Database consists of a plasma concentration versus time data from various drugs collected from the literature to highlight the variability in pharmacokinetic parameters for different drugs. It also contains data from real patients collected from HPJ to highlight the effects of age and health conditions on pharmacokinetic parameters of patients taking gentamicin and vancomycin. Clicking on data within the database from within a module will automatically transfer information into the corresponding text fields ready for calculation. Clicking the 'Submit' button will open the output window and show the corresponding graphs and pharmacokinetic parameters.

The CAL Module has two main functions. The Learning function contains several e-learning modules covering several topics in the fundamentals of pharmacokinetics and the basics of TDM. The Quiz and Games function contains quizzes and interactive scenario games. The quiz allows the user to test their knowledge in TDM and pharmacokinetics while the interactive games simulate a clinical setting

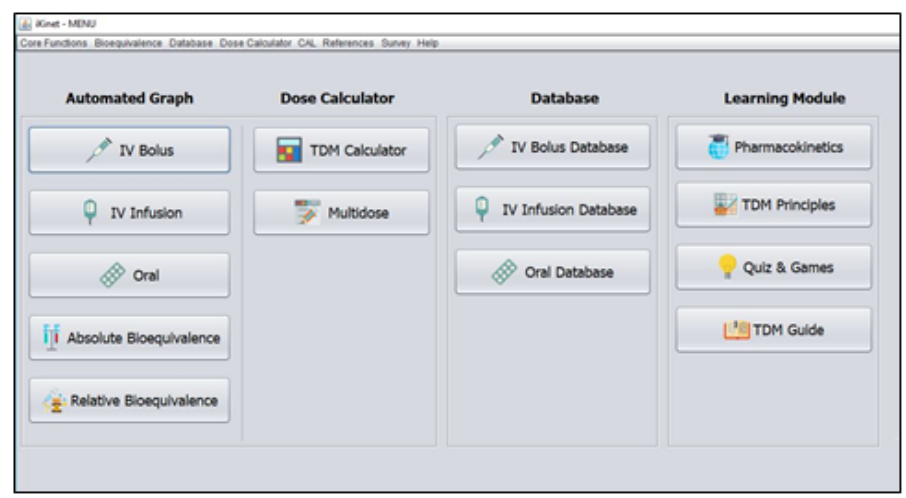

Figure 1: Software Main Menu.

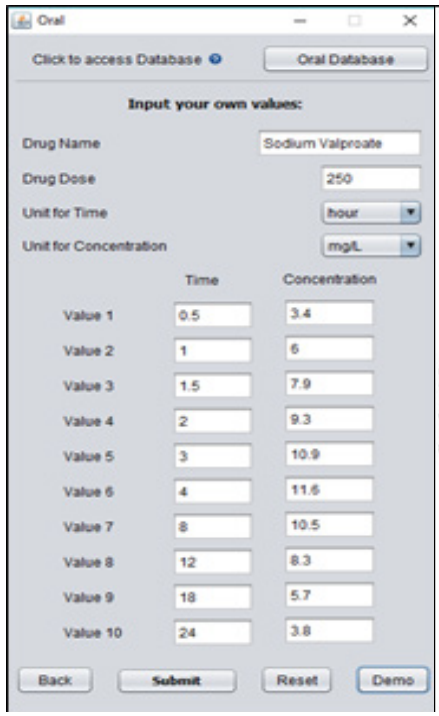

A. Oral dose Input Window

Figure 2: Input and Output Windows for Oral Dose Module.

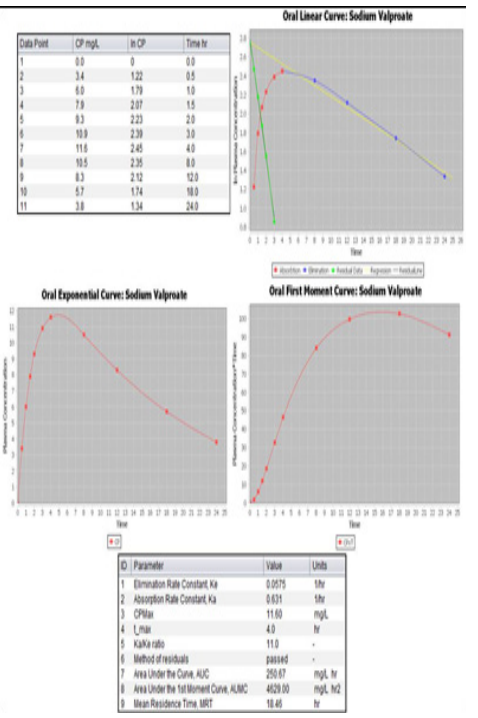

B. Oral dose output Window 
in which the user is a clinical pharmacist who is given the task to provide a suitable dosing regimen to a patient.

The TDM Calculator consists of a specialized calculator to adjust dose based on measured drug concentration. TDM Calculator focuses on specific drugs: aminoglycosides, vancomycin, phenytoin, valproate, carbamazepine, theophylline, digoxin and immunosuppressants. A multidose module is used to calculate the maintenance and loading dose of drugs that follow first-order pharmacokinetics, following IV bolus and oral administration. The main GUI window for the TDM Calculator contains a tabbed window with a 'calculator' that can be used for dosage adjustment of eight different drugs that are commonly indicated for TDM: aminoglycosides, vancomycin, phenytoin, sodium valproate, carbamazepine, theophylline, digoxin and immunosuppressants. Each drug can be accessed by clicking on its respective tab. All calculations are done based on the Malaysian Ministry of Health's (MOH) TDM guidelines. All fields must be filled and an empty field will trigger an error message. The aminoglycosides calculator can be used for gentamicin, amikacin and tobramycin, while the immunosuppressants calculator can be used for cyclosporine and tacrolimus.

\section{DISCUSSION}

Unit testing was conducted for each function in the Software using specifically prepared test cases to check accuracy. After completing unit testing, the testing process moved on to integration testing, specifically to ensure the correct flow of information between modules and the database. System testing was conducted with the help of a third party using a test case prepared with the assistance of a certified software tester and a registered pharmacist with prior experience in software testing. No major errors were discovered at this stage.

Validation of the calculations from modules involved comparing calculated parameters with manual calculations and graphs generated using Microsoft Excel. For manual calculations, the graphs were manually drawn and the graphs gradients and intercepts were determined and used for further calculation. The answers obtained from the relative bioavailability module are shown in Table 2 . It was noted that the values obtained from the Software calculations and Microsoft Excel matched each other well, but there were slight differences between the output and the answers obtained by manual calculations, particularly in the Oral Module (not shown). This is expected, however, as the margin of user

Table 2: Comparison of Calculations between iKinet, Excel and Manual Method for Relative Bioequivalence Module for Rifampicin. All units omitted for clarity.

\begin{tabular}{cccc}
\hline Parameters & iKinet & Excel & Manual Calculation \\
\hline $\mathrm{K}_{\mathrm{e}}$ Oral 1 & 0.240 & 0.240 & 0.243 \\
$\mathrm{~K}_{\mathrm{e}}$ Oral 2 & 0.236 & 0.236 & 0.235 \\
AUC Oral 1 & 54.63 & 54.63 & 54.59 \\
AUC Oral 2 & 58.32 & 58.32 & 58.33 \\
AUMC Oral 1 & 253.99 & 253.99 & 253.18 \\
AUMC Oral 2 & 286.57 & 286.57 & 286.75 \\
MRT Oral 1 & 4.65 & 4.65 & 4.64 \\
MRT Oral 2 & 4.91 & 4.91 & 4.92 \\
$\begin{array}{c}\text { Relative } \\
\text { bioavailability }\end{array}$ & 0.937 & 0.937 & 0.936 \\
\hline
\end{tabular}

error, when drawing graphs and manually determining absorption rate constants using the 'method of residuals' may be significant.

For the final validation step, multiple values of $\mathrm{K}_{\mathrm{e}}$ were compared between those from the Software, Microsoft Excel and manually calculated values and the second set of data compared the values produced with calculations made by HPJ pharmacists for the TDM modules. The first set of data compared 14 values of $\mathrm{K}_{\mathrm{e}}$ produced by the Automated Graph Modules against the values obtained by Microsoft Excel and manual method (Table 3). The second set of data compared 20 values of $\mathrm{K}_{\mathrm{e}}$ produced by the Automated Calculation Module and calculations made by HPJ pharmacists. 10 values were randomly selected from the Gentamicin Database and 10 values from the Vancomycin Database using an online randomizer [94]. The values of $\mathrm{K}_{\mathrm{e}}$ produced completely matched. A small variability can be observed from the comparison with manual calculations. This is expected when manually determining the line of best fit' on plotted points and the gradient of a graph. In any case, an analysis was performed to ensure that the differences between the output of the Software and manual calculation are not statistically significant. For comparison, three types of analysis were performed: correlation analysis, paired sample $t$-test and Bland Altman analysis. From the graph in Figure 3A, we can see that there is a linear correlation, with the Pearson coefficient value of $0.999(p<0.001)$, indicating statistical significance. Paired sample t-test analysis of the values of $K_{e}$ indicates a non-statistically significant difference between the two groups, $p=0.283$. The Bland Altman plot, which is illustrated in Figure $3 \mathrm{~B}$ also indicates non-significant difference between the two methods (Mean $=0.003$, SD $=0.01, p=0.283$ ). This shows that there is no statistically significant difference between the different output methods and the value of any difference is also extremely small.

170 participants were involved in software testing and a total of 116 completed responses were received. As expected, all respondents confidently expressed their computer skills. $42.86 \%$ of respondents considered themselves to be 'very good', followed by $42.06 \%$ and $15.08 \%$ for 'good' and 'average' categories respectively. Thus none of the respondents considered themselves to be lacking in computer skills. Once the student responses were converted into a scoring system, with a maximum score of 100 , with 68 being the cut-off point, ${ }^{19}$ a mean usability score of 68.8 (SD: 11.3) was obtained, which is just above

Table 3: Comparison of Elimination rate Calculations between iKinet, Excel and Manual Method for Numerous Drugs. All units omitted for clarity.

\begin{tabular}{ccccc}
\hline Module & Drug & iKinet & Excel & Manual \\
\hline IV Bolus & Methylprednisolone & 0.189 & 0.189 & 0.188 \\
IV Bolus & Rifampin & 0.252 & 0.252 & 0.253 \\
IV Infusion & Acyclovir & 0.773 & 0.773 & 0.774 \\
IV Infusion & Cefotaxime & 0.737 & 0.737 & 0.729 \\
Oral & Sodium Valproate & 0.0575 & 0.0575 & 0.0625 \\
Oral & Hydrochlorothiazide & 0.188 & 0.188 & 0.189 \\
Absolute BE & Ciprofloxacin Oral & 0.118 & 0.118 & 0.119 \\
Absolute BE & Ciprofloxacin IV & 0.121 & 0.121 & 0.121 \\
Absolute BE & Procainamide Oral & 0.245 & 0.245 & 0.254 \\
Absolute BE & Procainamide IV & 0.212 & 0.212 & 0.212 \\
Relative BE & Rifampicin 1 (Oral) & 0.24 & 0.24 & 0.243 \\
Relative BE & Rifampicin 2 (Oral) & 0.236 & 0.236 & 0.235 \\
Relative BE & Ampicillin 1 (Oral) & 0.514 & 0.514 & 0.549 \\
Relative BE & Ampicillin 2 (Oral) & 0.479 & 0.479 & 0.474 \\
\hline
\end{tabular}


Table 4: Summary of Responses for iKinet User Satisfaction Survey.

\begin{tabular}{|c|c|c|c|c|c|c|}
\hline \multirow[t]{2}{*}{ Question } & \multicolumn{2}{|c|}{ Strongly Agree } & \multicolumn{3}{|c|}{ Strongly Disagree } & \multirow[t]{2}{*}{ Tota } \\
\hline & 5 & 4 & 3 & 2 & 1 & \\
\hline 1. iKinet has a visually pleasing user interface & $\begin{array}{c}29 \\
(25.0 \%)\end{array}$ & $\begin{array}{c}72 \\
(62.1 \%)\end{array}$ & $\begin{array}{c}13 \\
(11.2 \%)\end{array}$ & $\begin{array}{c}1 \\
(0.9 \%)\end{array}$ & $\begin{array}{c}1 \\
(0.9 \%)\end{array}$ & 116 \\
\hline $\begin{array}{l}\text { 2. The output (eg: calculation and graph) provided by } \\
\text { iKinet are adequate for my needs }\end{array}$ & $\begin{array}{c}45 \\
(38.8 \%)\end{array}$ & $\begin{array}{c}60 \\
(51.7 \%)\end{array}$ & $\begin{array}{c}9 \\
(7.8 \%)\end{array}$ & $\begin{array}{c}1 \\
(0.9 \%)\end{array}$ & $\begin{array}{c}1 \\
(0.9 \%)\end{array}$ & 116 \\
\hline $\begin{array}{l}\text { 3. The output (eg: graphs and tables) produced by iKinet } \\
\text { are easy to understand }\end{array}$ & $\begin{array}{c}44 \\
(37.9 \%)\end{array}$ & $\begin{array}{c}62 \\
(53.4 \%)\end{array}$ & $\begin{array}{c}6 \\
(5.2 \%)\end{array}$ & $\begin{array}{c}3 \\
(2.6 \%)\end{array}$ & $\begin{array}{c}1 \\
(0.9 \%)\end{array}$ & 116 \\
\hline $\begin{array}{l}\text { 4. I think that using iKinet will enhance my understanding } \\
\text { of pharmacokinetics }\end{array}$ & $\begin{array}{c}52 \\
(44.8 \%)\end{array}$ & $\begin{array}{c}52 \\
(44.8 \%)\end{array}$ & $\begin{array}{c}9 \\
(7.8 \%)\end{array}$ & $\begin{array}{c}2 \\
(1.7 \%)\end{array}$ & $\begin{array}{c}1 \\
(0.9 \%)\end{array}$ & 116 \\
\hline $\begin{array}{l}\text { 5. I would recommend iKinet to my friends/colleagues } \\
\text { who want to learn pharmacokinetics }\end{array}$ & $\begin{array}{c}56 \\
(48.3 \%)\end{array}$ & $\begin{array}{c}52 \\
(44.8 \%)\end{array}$ & $\begin{array}{c}7 \\
(6.0 \%)\end{array}$ & $\begin{array}{c}0 \\
(0.0 \%)\end{array}$ & $\begin{array}{c}1 \\
(0.9 \%)\end{array}$ & 116 \\
\hline 6. The modules are easy to understand & $\begin{array}{c}45 \\
(38.8 \%)\end{array}$ & $\begin{array}{c}59 \\
(50.9 \%)\end{array}$ & $\begin{array}{c}9 \\
(7.8 \%)\end{array}$ & $\begin{array}{c}2 \\
(1.7 \%)\end{array}$ & $\begin{array}{c}1 \\
(0.9 \%)\end{array}$ & 116 \\
\hline 7. The modules flow smoothly from one topic to another & $\begin{array}{c}39 \\
(33.6 \%)\end{array}$ & $\begin{array}{c}62 \\
(53.4 \%)\end{array}$ & $\begin{array}{c}13 \\
(11.2 \%)\end{array}$ & $\begin{array}{c}1 \\
(0.9 \%)\end{array}$ & $\begin{array}{c}1 \\
(0.9 \%)\end{array}$ & 116 \\
\hline $\begin{array}{l}\text { 8. The visuals and fonts from the modules are pleasant } \\
\text { and appropriate }\end{array}$ & $\begin{array}{c}34 \\
(29.3 \%)\end{array}$ & $\begin{array}{c}69 \\
(59.5 \%)\end{array}$ & $\begin{array}{c}10 \\
(8.6 \%)\end{array}$ & $\begin{array}{c}2 \\
(1.7 \%)\end{array}$ & $\begin{array}{c}1 \\
(0.9 \%)\end{array}$ & 116 \\
\hline $\begin{array}{l}\text { 9. The sound and voice used in the modules are clear and } \\
\text { easy to understand }\end{array}$ & $\begin{array}{c}32 \\
(27.6 \%)\end{array}$ & $\begin{array}{c}55 \\
(47.4 \%)\end{array}$ & $\begin{array}{c}23 \\
(19.8 \%)\end{array}$ & $\begin{array}{c}3 \\
(2.6 \%)\end{array}$ & $\begin{array}{c}3 \\
(2.6 \%)\end{array}$ & 116 \\
\hline 10. The quiz questions within the modules are relevant & $\begin{array}{c}42 \\
(36.2 \%)\end{array}$ & $\begin{array}{c}63 \\
(54.3 \%)\end{array}$ & $\begin{array}{c}9 \\
(7.8 \%)\end{array}$ & $\begin{array}{c}2 \\
(1.8 \%)\end{array}$ & $\begin{array}{c}0 \\
(0.0 \%)\end{array}$ & 116 \\
\hline
\end{tabular}
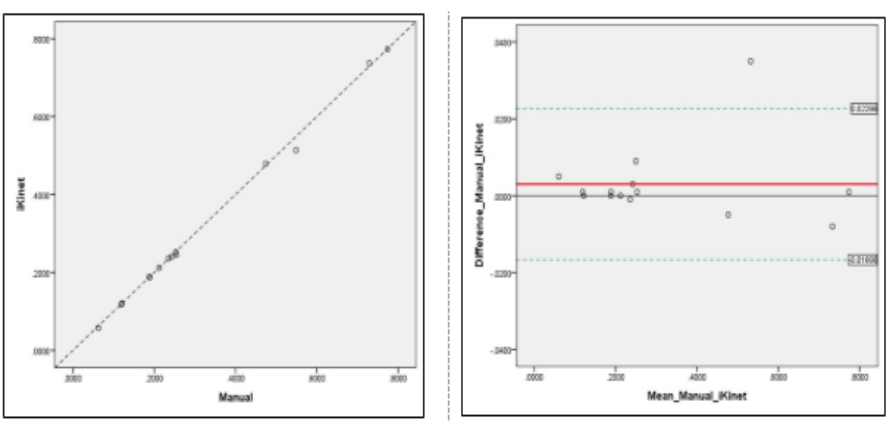

Figure 3: Comparison of Output from the Software and Manual Calculation Methods.

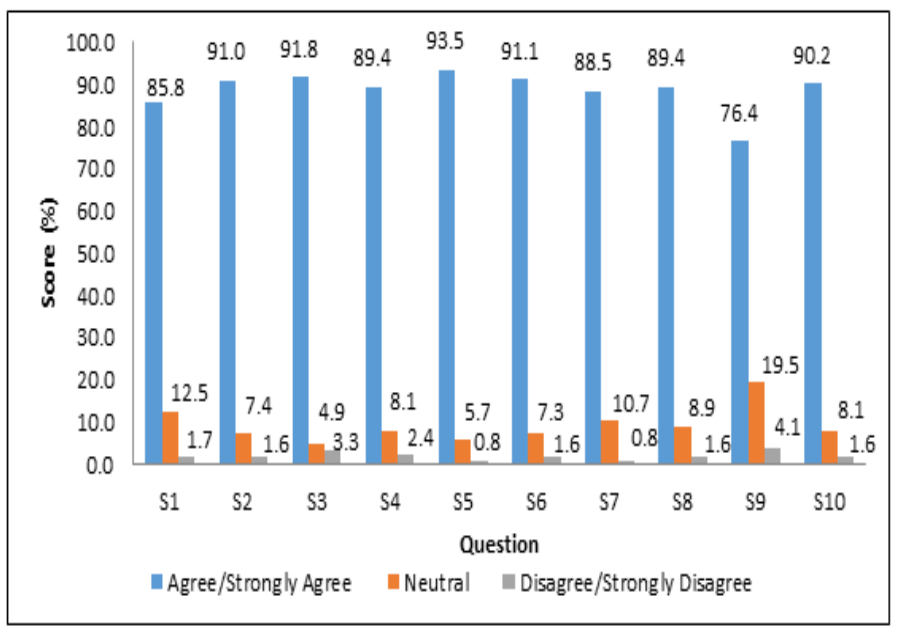

Figure 4: Bar Chart Depicting Positive, Neutral and Negative Opinions from the User Satisfaction Survey. that deemed acceptable. Although this score is considered acceptable, student response will be utilized to further refine the software's graphical user interface (GUI). The next iteration of the system will be web-based, allowing for better user experience. A summary of the responses is presented in Table 4 . When grouped for comparison (Figure 4), there is a significant difference between positive opinions and negative or neutral opinions, with the majority of respondents expressing satisfaction. More than $80 \%$ of respondents expressed satisfaction in nine of the 10 items, except item number 9, which assessed satisfaction on the voices and sounds used in the CAL modules.

\section{CONCLUSION}

A modular pharmacokinetics computer program was successfully developed using Java and the NetBeans IDE Version 8.2. Java was chosen as it allows portability between devices, where the same source code can be used for different operating systems including Windows, Mac OS and Android. Consisting of three main components: An Automated Calculation Module, a Pharmacokinetic Database and a CAL Module. Each was structured to assist students to understand pharmacokinetics; from content delivery, calculation assistance, a database to give access to various pharmacokinetic profiles and student-based activities for teaching and learning. Three levels of software testing were completed: unit testing, integration testing and system testing. A user satisfaction directed at mainly third-year pharmacy undergraduates revealed an average usability score of 68.8 , indicating good usability. The end-users were generally satisfied with the software in its current state and provided good feedback and recommendations for further improvements. The next steps are to further optimize the program and to introduce the software package to students during undergraduate teaching, in an attempt to augment lectures and tutorials and allow students to gain a visual validation of their manual calculations 


\section{ACKNOWLEDGEMENT}

The authors would like to thank the Institute of Research Management and Innovation, UiTM (600-IRMI/MyRA 5/3/SINERGI 011/2018) for financial support.

\section{CONFLICT OF INTEREST}

The authors declare that there is no conflict of interest.

\section{ABBREVIATIONS}

AUC: Area Under the Curve; AUMC: Area Under the First Moment Curve; CAL: Computer Aided Learning; GUI: Graphical User Interface; IDE: Integrated Development Environment; IV: Intravenous; PRP: Provisionally Registered Pharmacist; TDM: Therapeutic Drug Monitoring; MOH: Ministry of Health; MAT: Mean Absorption Time; MRT: Mean Residence Time; HPJ: Hospital Putra Jaya; NMRR: National Medical Research Register; REC: Research Ethics Committee; Ke: Elimination Rate Constant; $\mathbf{t}_{\mathbf{1} / 2}:$ Half-life; Vd: Volume of Distribution.

\section{REFERENCES}

1. Ye ZK, Tang HL, Zhai SD. Benefits of therapeutic drug monitoring of vancomycin: A systematic review and meta-analysis. PLoS One. 2013;8(10):1-10.

2. Pearce GA, McLachlan AJ, Ramzan I. Bioequivalence: How, Why and What Does it Really Mean?. J Pharm Pract Res. 2004;34(3):195-200.

3. Samsiah A, Othman N, Jamshed S, Hassali MA, Wan-Mohaina WM. Medication errors reported to the National Medication Error Reporting System in Malaysia: A 4-year retrospective review (2009 to 2012). Eur J Clin Pharmacol. 2016;72(12):1515-24.

4. Warner AM. Pitfalls in Monitoring Therapeutic Drugs. Lab Med. 1997; 28(10):653-7.

5. Persky AM. Multi-faceted approach to improve learning in pharmacokinetics. Am J Pharm Edu. 2008;72(2):36-9.

6. Persky AM. The impact of team-based learning on a foundational pharmacokinetics course. Am J Pharm Edu. 2012;76(2):31-6.

7. Persky AM, Stegall-Zanation J, Dupuis RE. Students perceptions of the incorporation of games into classroom instruction for basic and clinical pharmacokinetics. Am J Pharm Edu. 2007;71(2):21-5.

8. Franklin AS, Markowsky S, DeLeo J, Normann S, Black E. Using Team-based Learning to Teach a Hybrid Pharmacokinetics Course Online and in Class. Am J Pharm Edu. 2016;80(10):171-5.

9. Munar MY, Singh H, Belle D, Brackett CC, Earle SB. The use of wireless laptop computers for computer-assisted learning in pharmacokinetics. Am J Pharm Edu. 2006;70(1):4-7.

10. Blouin RA, Rifee WH, Robinson ET, Beck DE, Green C, Joyner PU, et al. Roles of Innovation in Education Delivery. Am J Pharm Edu. 2009:73(8):154-9.

11. Deloitte Global Services Limited. Younger Millennials: The Pro-PC Generation. The Wall Street Journal. 2016. Available: http://deloitte.wsj.com/cio/2016/04/21/ younger-millennials-the-pro-pc-generation/ [Accessed: July 29, 2018].

12. Björkman S. Evaluation of the TCIWorks Bayesian computer program for estimation of individual pharmacokinetics of FVIII. Haemophilia. 2011;1(17):23940.

13. Yung-jin L, Jian-ming L, Ying-hao L. Javba PK for Deskltop. 2018. Available from: http://pkpd.kmu.edu.tw/jpkd/about.htm Bourne DWA. Pharmacokinetic Software. 2017. Available from: https://www.pharmpk.com/soft.html Krejcie RV Morgan DW. Determining Sample Size for Research Activities. Educational and Psychogical Measurement. 1970;30(3):607-10.

14. The Malaysian Administrative Modernisation and Management Planning Unit (MAMPU). Portal Data Terbuka Malaysia. 2018. Available from: http://www. data.gov.my/data/ms_MY/dataset Urbaniak GC, Plous S. Research Randomizer. 2018. Available from: https://www.randomizer.org/ [Accessed: May 10, 2018]

15. Giavarina D. Understanding Bland Altman Analysis. Biochemia Medica. 2015;25(2):141-51.

16. Brooke J. SUS: A Quick and dirty usability scale. Usability Evaluation in Industry. Taylor and Francis. 1996.

17. LaLomia MJ Sidowski JB. Measurements of computer satisfaction, literacy and aptitudes: A review. International Journal of Human-Computer Interaction. 1990;2(3):231-53.

18. Laugwitz B, Held T, Schrepp M. Construction and Evaluation of a User Experience Questionnaire. $\mathrm{HCl}$ and Usability for Education and Work USAB, Graz: Austria. 2008;63-76.

19. Pallant JF. SPSS Survival Manual: A Step by Step Guide to Data Analysis using IBM SPSS, $3^{\text {rd }}$ ed. Open University Press. 2007.

Article History: Submission Date : 19-07-2020; Revised Date : 29-08-2020; Acceptance Date : 05-10-2020

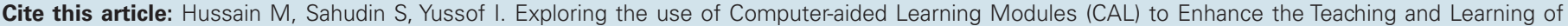
Pharmacokinetics to Pharmacy Students. J Young Pharm. 2020;12(4):354-9. 